\title{
Reply to: "An appeal to cost undermines food security risks of delayed mitigation"
}

\section{Authors:}

Tomoko Hasegawa ${ }^{1,2,3^{*}}$, Shinichiro Fujimori²,3,4, Petr Havlík², Hugo Valin², Benjamin Leon Bodirsky ${ }^{5}$, Jonathan C. Doelman ${ }^{6}$, Thomas Fellmann, Page Kyle ${ }^{8}$, Jason F. L. Koopman ${ }^{9}$, Hermann Lotze-Campen ${ }^{5,10}$, Daniel Mason-D'Croz ${ }^{11,12}$, Christoph Müller ${ }^{5}$, Yuki Ochi ${ }^{13}$, Ignacio Pérez Domínguez ${ }^{7}$, Elke Stehfest ${ }^{6}$, Timothy B. Sulser ${ }^{11}$, Andrzej Tabeau $^{9}$, Kiyoshi Takahashi ${ }^{3}$, Jun'ya Takakura ${ }^{3}$, Hans van Meij19,14, Willem-Jan van Zeist $^{6}$, Keith Wiebe ${ }^{11}$, Peter Witzke ${ }^{15}$

\section{Author Affiliations:}

1. Ritsumeikan University, 1-1-1, Nojihigashi, Kusatsu, Shiga, 525-8577, Japan.

2. International Institute for Applied System Analysis (IIASA), Schlossplatz 1, A2361 Laxenburg, Austria

3. Center for Social and Environmental Systems Research, National Institute for Environmental Studies (NIES), 16-2 Onogawa, Tsukuba, Ibaraki 305-8506, Japan

4. Department of Environmental Engineering, Graduate School of Engineering, Kyoto University, C1-3, Kyoto Daigaku Katsura, Nishikyo-ku, Kyoto-shi, 615-8540, Japan.

5. Potsdam Institute for Climate Impact Research (PIK), Telegrafenberg A 31, 14473, Potsdam, Germany

6. PBL Netherlands Environmental Assessment Agency, Postbus 30314, 2500 GH The Hague, The Netherlands

7. European Commission, Joint Research Centre, c/Inca Garcilaso 3, 41092 Seville, Spain

8. Joint Global Change Research Institute, Pacific Northwest National Laboratory, 5825 University Research Court, Suite 3500, College Park, MD 20740, US

9. Wageningen Economic Research, Wageningen University and Research, 2585 DB The Hague, Netherlands

10. Humboldt-Universität zu Berlin, 10099 Berlin, Germany

11. International Food Policy Research Institute (IFPRI), 1201 I St. NW, Washington, DC 20005, USA

12. Commonwealth Scientific and Industrial Research Organisation (CSIRO), 306 Carmody Rd, St Lucia QLD 4067, Australia

13. E-Konzal Co. Ltd, 3-8-15, Nishinakajima, Yodogawa, Osaka, 532-0011, Japan 
14. Agricultural Economics and Rural Policy Group, Wageningen University, Hollandseweg 1, 6706 KN Wageningen, the Netherlands

15. Institute for Food and Resource Economics, University of Bonn, Nussallee 21, D53115 Bonn, Germany

* To whom correspondence should be addressed: thase@fc.ritsumei.ac.jp

\section{Main text:}

The core of the critique by Hayek at al. (2019) ${ }^{1}$ of our paper ${ }^{2}$ seems to be that by raising concerns about secondary impacts of emissions mitigation efforts, our study will hinder social and political efforts to reduce emissions. However, this is contrary to what we intend; to quote from the study: "In particular, it highlights the need for carefully designed mitigation policies for agriculture and land use, to ensure that progress towards climate stabilization and food security can be simultaneously achieved." Nowhere in our paper do we suggest that delaying mitigation efforts is an option for the future.

Hayek et al. (2019) claim that our study is based on an inappropriate and opaque set of model ensembles and assumptions. While we understand concerns that using a large number of complex models does inherently reduce the transparency and replicability of the research, each of the models used is individually well-documented and established, and together these models have already been used in a number of published intercomparisons on both agricultural climate impacts, and emissions mitigation. Our modelling approach, scenario settings and assumptions reflect state-of-the-art methods of modelling and analysis of these topics. Although all models have limitations, and our scenarios do not reflect either the full suite of future climate-related risks, nor all policy strategies for emissions mitigation, the study does offer valid, relevant insights into the complex nature of climate change impacts and mitigation.

(1) Hayek et al. (2019) raised five arguments, which we will address in turn. They first challenge that our model assessment is based on climatic means and does not represent the full suite of risks that climate change poses directly to agriculture, such as the impacts of extreme climate events. For clarification of the methods used, we used daily values of temperature and precipitation from climate models, biascorrected and downscaled to 0.5 degree resolution, to drive process-based global 
gridded crop growth models. Resulting yield changes by crop and nation were averaged (30-year means) and provided to economic models with coarser temporal and (in most cases) geographic resolution ${ }^{3}$. We acknowledge that the approach misses several types of extreme events (e.g., hail, storm damage), and buffers interannual variability and the consequences thereof. Still, the approach reflects the capabilities of the economic models as a group. To represent a more complete picture of the threat of climate change on the agricultural sector, future improvement in economic models should address inter-annual variability in crop yields, stocks, and adaptation barriers, among other features.

(2) The second concern is that our study does not consider several other impacts of climate change through ozone pollution, pollination declines, or sea level rise. To represent a more complete picture of climate change impacts on the agricultural sector including these aforementioned aspects, future studies are needed. However, the effects of ozone on agricultural climate impacts are ambiguous, as indicated in the cited paper ${ }^{4}$. Moreover, the impacts of sea level rise on food production would likely be limited at a global level, though it may significantly affect some regions. Accordingly, assessment of regional food security should consider such impacts. While the impacts of climate change on food production through pollination disturbance have been suggested by some studies recently, quantitative analyses at global scale has not yet been available.

(3) The third concern is that our findings were previously identified by earlier studies. However, the earlier studies mentioned by Hayek et al. were based on a single model. As we documented, the models are heterogeneous in structure, baseline scenario results, and climate impact responses. Increasing the number of models offers a more comprehensive picture of the research topics considered, thereby improving upon the existing literature.

(4) The fourth concern is that our assessment does not include taxation of the indirect emissions from meat production; in fact, our study does consider indirect GHG emissions from meat production. As indicated in the original paper, the assumed price on agricultural GHG emissions increases production costs according to modeled GHG emission intensity. This includes crops used as feed; livestock producers must therefore pay for the indirect GHG emissions from feed production. In the scenarios, the GHG emissions prices lead to increased prices and decreased dietary 
consumption of both crop and livestock products, and also lead to a shift in the composition of the consumer diet from animal products to crop-based products, alleviating negative effects on global food security. However, the degrees to which such changes in production, consumption, and prices are observed in the models reflect that (i) crops and animal products are not perfect dietary substitutes, and have differentiated price elasticities; and (ii) the future GHG emissions intensity is not a fixed characteristic of either crop or animal commodity production.

(5) Finally, the fifth concern is that a uniform carbon tax does not reflect realistic policies and is not in the spirit of Article 3 of the UNFCCC; that we implemented it for utility and parsimony in modelling rather than for efficacy or fairness of the envisaged policy. As is common practice in the integrated assessment modeling literature, we implemented the climate change mitigation targets by putting a global uniform GHG emissions price across all sectors and regions. Rather than representing the complexities of policy-making, global uniform carbon prices are used to represent economically efficient mitigation and its distribution across sectors, regions, and time. We selected the approach not only for utility and parsimony in modelling but also for efficiency and efficacy. We acknowledge that such a policy can have undesirable aspects, such as impacts on vulnerable populations. Still, exclusion of selected regions and/or sectors from the policy is known to require larger and often very costly emissions reductions from the remainder of the system to reach overall climate targets, and for ambitious mitigation targets, such exemptions may put the mitigation goals out of reach ${ }^{5}$. We agree that in reality, a wide range of policies has been implemented and discussed for the land-use-related sectors, including investment in research and development, subsidies for adoption of GHG-efficient agricultural practices, and supplementary policies to target food security ${ }^{6}$. In this respect, further research is needed to identify policy packages that could achieve climate mitigation targets while avoiding the critical finding of our study.

Here, we want to re-emphasize that our findings on food security concerns should not be used to delay emissions mitigation, but rather that mitigation efforts need to consider possible unintended consequences. In this sense our article ${ }^{2}$ cautions against overly simplistic implementation of climate mitigation policies and highlights the need for differentiated, targeted solutions for agriculture and complementary measures for food security. 
Reference

1. Hayek MN, McDermid SP, Jamieson DW. An appeal to cost undermines food security risks of delayed mitigation. Nature Climate Change 2019.

2. Hasegawa T, Fujimori S, Havlík P, Valin H, Bodirsky BL, Doelman JC, et al. Risk of increased food insecurity under stringent global climate change mitigation policy. Nature Climate Change 2018, 8(8): 699-703.

3. Müller C, Robertson RD. Projecting future crop productivity for global economic modeling. Agricultural Economics 2014, 45(1): 37-50.

4. Tai APK, Martin MV, Heald CL. Threat to future global food security from climate change and ozone air pollution. Nature Climate Change 2014, 4(9): 817-821.

5. Clarke L. KJ, K. Akimoto, M. Babiker, G. Blanford, K. Fisher-Vanden, J.-C. Hourcade, V. Krey, E. Kriegler, A. Löschel, D. McCollum, S. Paltsev, S. Rose, P.R. Shukla, M. Tavoni, B.C.C. van der Zwaan, and D.P. van Vuuren. Assessing Transformation Pathways. In: Edenhofer O, R. Pichs-Madruga, Y. Sokona, E. Farahani, S. Kadner, K. Seyboth, A. Adler, I. Baum, S. Brunner, P. Eickemeier, B. Kriemann, J. Savolainen, S. Schlömer, C. von Stechow, T. Zwickel and J.C. Minx (ed). Climate Change 2014: Mitigation of Climate Change. Contribution of Working Group III to the Fifth Assessment Report of the Intergovernmental Panel on Climate Change. Cambridge University Press: Cambridge, United Kingdom and New York, NY, USA, 2014.

6. Doelman JC, Stehfest E, Tabeau A, van Meijl H. Making the Paris agreement climate targets consistent with food security objectives. Global Food Security 2019, 23: 93103. 